\title{
Simple equations to predict concentric lower-body muscle power in older adults using the 30-second chair-rise test: a pilot study
}

This article was published in the following Dove Press journal:

Clinical Interventions in Aging

3 July 2010

Number of times this article has been viewed

\author{
Wesley N Smith' \\ Gianluca Del Rossi' \\ Jessica B Adams' \\ KZ Abderlarahman ${ }^{2}$ \\ Shihab A Asfour ${ }^{2}$ \\ Bernard A Roos 1,3,4,5 \\ Joseph F Signorile ${ }^{1,3}$
}

'Department of Exercise and Sport Sciences, ${ }^{2}$ Department of Industrial Engineering, University of Miami, Coral Gables, FL, USA; ${ }^{3}$ Geriatric Research, Education, and Clinical Center, Bruce W Carter Department of Veterans Affairs Medical Center, Miami, FL, USA; ${ }^{4}$ Departments of Medicine and Neurology, University of Miami Miller School of Medicine, Miami, FL, USA; ${ }^{5}$ Stein Gerontological Institute, Miami Jewish Health Systems, Miami, FL, USA

Correspondence: Joseph F Signorile University of Miami, PO Box 248065, Coral Gables, FL 33 I 24, USA

$\mathrm{Tel}+\mid 305898$ |457

$\mathrm{Fax}+\mathrm{I} 3052845168$

Email jsignorile@miami.edu

\begin{abstract}
Although muscle power is an important factor affecting independence in older adults, there is no inexpensive or convenient test to quantify power in this population. Therefore, this pilot study examined whether regression equations for evaluating muscle power in older adults could be derived from a simple chair-rise test. We collected data from a 30-second chair-rise test performed by fourteen older adults (76 \pm 7.19 years). Average (AP) and peak (PP) power values were computed using data from force-platform and high-speed motion analyses. Using each participant's body mass and the number of chair rises performed during the first 20 seconds of the 30-second trial, we developed multivariate linear regression equations to predict AP and PP. The values computed using these equations showed a significant linear correlation with the values derived from our force-platform and high-speed motion analyses (AP: $\mathrm{R}=0.89$; $\mathrm{PP}: \mathrm{R}=0.90$; $P<0.01)$. Our results indicate that lower-body muscle power in fit older adults can be accurately evaluated using the data from the initial 20 seconds of a simple 30 -second chair-rise test, which requires no special equipment, preparation, or setting.
\end{abstract}

Keywords: instrumental activity of daily living, clinical test, elderly, chair-stand test, leg power

\section{Introduction}

The mechanical power produced by skeletal muscle, especially as it relates to the repetitive movements performed during daily activities, may be the most significant physical factor affecting mobility, fall risk, and functional debility in older individuals.$^{1-3}$ In fact, muscle power affects activities of daily living (ADLs) and instrumental activities of daily living (IADLs) more than strength.,

Physical assessment of skeletal muscle power can help predict functional decline, loss of independence, and even frailty. Among traditional tests for measuring power are the vertical jump on a force platform, computerized cycle ergometry, isokinetic dynamometry, and computer-interfaced rigs specifically modified to calculate power output. $^{6-19}$ However, these tests are rarely used in clinical settings because of the expense involved and the requirements for specific, nonportable technology and software, and often, trained personnel to run the tests.

Field tests including stair-climbing, ramp-ascent, and vertical-jump tests can also assess muscular power in elderly individuals. Both ramp- and stair-climbing tests have been shown to be reliable tests of power, ${ }^{16,20}$ but inconvenient, since patients generally must leave the examination room for testing. These tests also have other drawbacks: the evaluation of power with ramp testing is specific to the slope of the ramp used, ${ }^{20}$ and stair-climbing tests can be adversely affected by an elderly person's fear of falling 
on the stairs. ${ }^{21}$ Jump tests differ biomechanically from typical ADLs, and they may pose a safety risk or be too challenging for older individuals.

Another consideration is the nature of the power to be measured. Clearly, the tests included in this introduction vary mechanically and metabolically. Jump tests represent a highly explosive form of mechanical power, while the metabolic determinants of stair or ramp tests, the cycle ergometry tests, and chair-rise test used in this study, reflect progressively lower levels of mechanical and metabolic power due to their testing duration. These more "prolonged" tests often allow the measurement of both peak and average power.

If clinicians could readily detect deficits in muscle absolute power and power per unit body weight, they might be able to prescribe exercise or other interventions to improve performance and reduce or even reverse physical vulnerability in older persons. ${ }^{11,22,23}$ Therefore, the purpose of this pilot study was to develop a regression equation that would quantify the lower-body skeletal muscle power of older adults via a simple chair-rise test. This field test and resulting predictive equation comprise a highly portable, cost-effective, and simple method enabling geriatricians and allied health professionals to predict lower-body muscle power in older men and women.

\section{Methods}

Participants were recruited through flyers posted at the University of Miami Wellness Center and the Aventura Jewish Community Center. Written informed consent approved by the university's Committee for the Use and Protection of Human Subjects was obtained from each participant in a face-to-face interview. Before acceptance into the study, potential participants completed a medical history questionnaire and were screened for resting heart rate and blood pressure. Inclusion criteria were: older than 65 years, living independently in the community, physician approval to participate, and willingness to perform the study protocol. Exclusion criteria were: a resting systolic blood pressure greater than $165 \mathrm{mmHg}$ or diastolic above $95 \mathrm{mmHg}$, a resting heart rate faster than $100 \mathrm{bpm}$ or slower than $50 \mathrm{bpm}$, self-reported symptoms of coronary or peripheral vascular disease since last physical examination, neuromuscular or musculoskeletal disorders disrupting voluntary movement, limb amputation, limb injury within the last three months, inability to speak or read English, and cognitive impairment, hernia, or hemorrhoids. Participant characteristics are presented in Table 1.
Table I Subject characteristics

\begin{tabular}{llll}
\hline & Women $(\mathrm{n}=10)$ & Men $(\mathrm{n}=4)$ & Total $(\mathrm{n}=14)$ \\
\hline Age $(\mathrm{y})$ & $75.7 \pm 6.8$ & $76.8 \pm 9.1$ & $76.0 \pm 7.2$ \\
Height $(\mathrm{m})$ & $1.61 \pm 0.71$ & $1.72 \pm 0.94$ & $1.64 \pm 0.99$ \\
Weight $(\mathrm{kg})$ & $64.8 \pm 14.7$ & $83.2 \pm 17.7$ & $70.0 \pm 17.2$ \\
\hline
\end{tabular}

Notes: All values are means \pm SD.

\section{Study design}

This prospective, observational study examined test data produced by men and women over 65 years of age with diverse levels of physical function. Movement velocity and ground reaction force data were collected during a 30 -second period of successive chair rises via a movement analysis system interfaced with a force plate. The number of chair rises, femur length (distance traveled), body mass, and gender were the preliminary variables in constructing predictive equations for peak power (PP) and average power (AP).

\section{Testing procedures}

Testing began with obtaining informed consent, taking resting measurements, and completing the medical history questionnaire. Measures were also taken of height, weight, and femur length from the greater trochanter to the lateral epicondyle.

\section{Lower-body muscle power measurement}

Lower-body muscle power was assessed during the 30 -second chair-rise test, ${ }^{24}$ which measures the number of stands from an armless chair of standard height $(45 \mathrm{~cm})$ performed in 30 seconds. Two 20.5-kg weights were placed behind the chair to restrict movement because the chair could not be placed against a wall without restricting the line-of-sight of the motion-analysis cameras.

Before data collection, the participant performed a threerepetition practice trial to become familiar with the test and to allow evaluation of his or her technique. The test began when the participant was in seated position with a neutral spine and feet flat on the floor. The participant was instructed to rise to a full stand and return to the original seated position as quickly as possible. The chair rises began with the participant's arms crossed at the wrist and held against the chest. The participant was verbally cued using the command "1, 2, 3, GO," and a stopwatch was started simultaneously with the "GO" cue. Participants were instructed to move at a maximal speed until they either felt the need to stop or the 30 -second time limit was reached. The initial trial was followed by a five-minute recovery and a second trial. 
Force was measured using a Kistler Instrument ${ }^{\complement}$ force platform (Amherst, NY). Cumulative forces from both the left and right legs were combined in the analysis. The platform was mounted so that its upper surface was level with the laboratory floor. Velocity was measured simultaneously with a high-speed photography system (Vicon, Lake Forest, CA).

The marker attachment protocol for the Vicon system was based on the plug-in gait marker set, in which a total of 48 reflective markers were attached at predetermined points on both the upper and lower body. The movement of these markers/body segments was recorded by a total of eight infrared cameras. The recording was made at a speed of 120 frames per second. The cameras recorded the X, Y, and $\mathrm{Z}$ coordinates' values at every time frame at the rate mentioned, then both the velocity and acceleration of joint centers and the different segments of interest were calculated by performing single and double differentiation.

The whole body center of mass, which represents the net result of the instantaneous limb movements and force production during a given activity, was determined by processing the 14-segment biomechanical full body model (head, trunk, two upper arms, two lower arms, two hands, two thighs, two shanks, and two feet), then the whole body center of mass was calculated using both the Vicion plug-in model as well as the Johan model. ${ }^{25}$ The motion-capturing data were filtered using a Woltring filtering routine.

Force and velocity data were transmitted through an analog-to-digital converter at 125 samples per second to a Dell Precision $650^{\circledR}$ high-performance workstation. Data were recorded and analyzed using Matlab Version 6 software (Mathworks Inc., Natick, MA). Power was calculated as the product of force production and simultaneous movement velocity during each stand.

Chair-rise power was computed during hip and knee extension, which started when the participant began rising from the seated position (after anterior pelvic tilt) and ended when the participant was standing upright. The beginning and end of chair-rise power were determined via start and end of positive velocity measured at the anterior superior iliac spine (ASIS) (Figure 1). In our laboratories we have performed over 100 repeated chair-stand tests with an ICC within a single day of $\mathrm{R}=0.93$ and across two non-successive days of $\mathrm{R}=0.91$. Unfortunately, only 8 subjects contributed data to our reliability analysis in the current study; however, for these subjects there was a within-day ICC of $\mathrm{R}=0.98$.

\section{Statistics}

The main response variables were the participant's combined right and left leg PP produced at any point during the test and

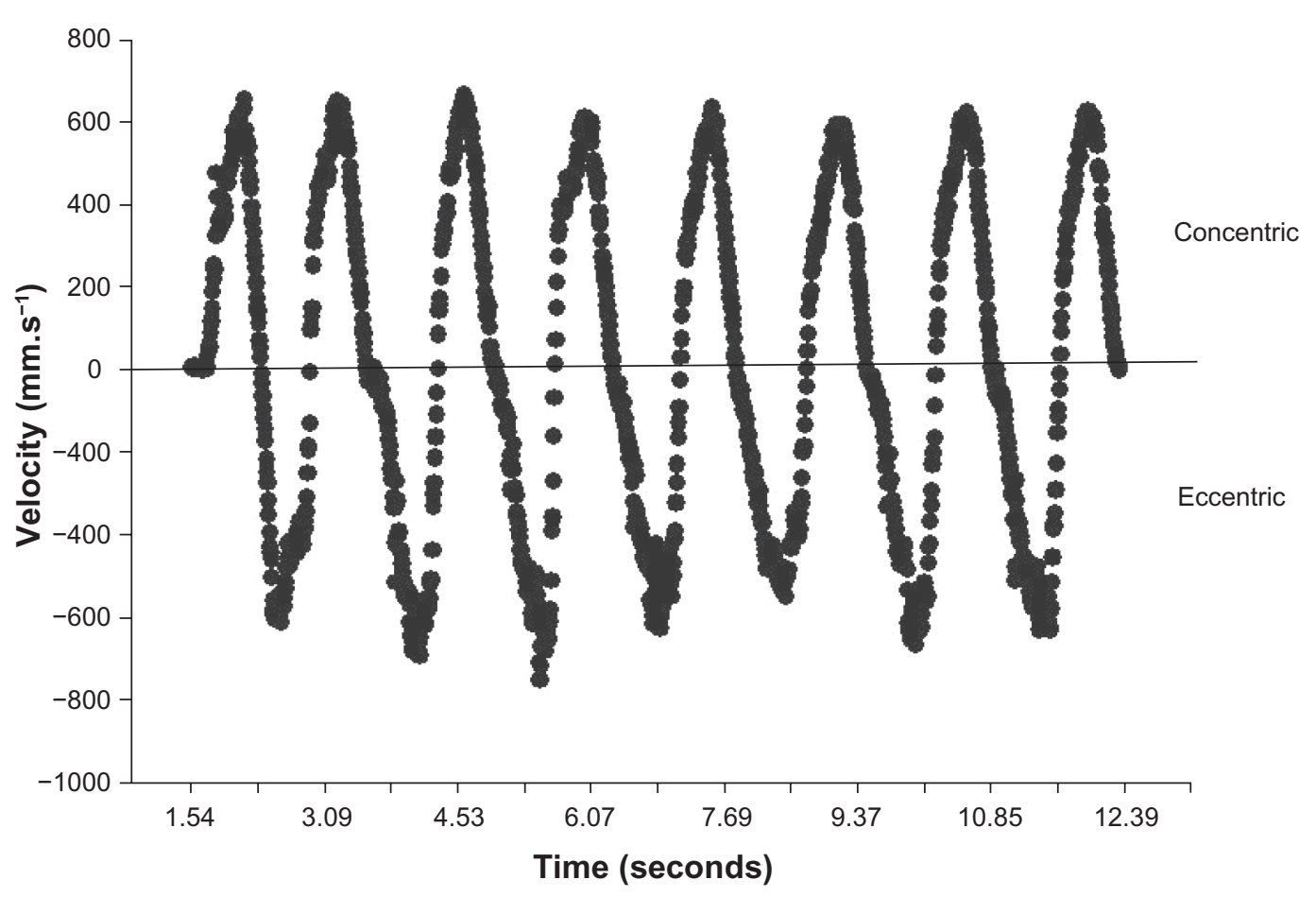

Figure I A randomly chosen representative sample of eight successive chair rises. Individual chair rises were distinguished via positive and negative velocity changes from zero at the anterior superior iliac spine. 
combined AP achieved per chair rise. Independent variables that served as parameters to predict the response variables were: 1) the number of complete chair rises performed in $10,15,20$, and 30 seconds; 2) the participant's weight in $\mathrm{kg}$; 3 ) the length of the participant's femur in $\mathrm{cm}$, from the greater trochanter to the lateral epicondyle, as a measure of the distance traveled during the chair rise; and 4) gender. A linear model was developed to estimate the peak power in watts achieved during the chair-rise test using the linear regression equation:

$$
\mathrm{y}=\mathrm{B}_{0}+\mathrm{B}_{1} \mathrm{X}_{1},+\mathrm{B}_{2} \mathrm{X}_{2},+\mathrm{B}_{3} \mathrm{X}_{3}+\mathrm{B}_{4} \mathrm{X}_{4}+\varepsilon
$$

where: $\mathrm{Y}$ is the peak power in watts; $\mathrm{X}_{1}$ represents the time it takes to complete the number of chair rises during the specified time period; $X_{2}$ is the subject's weight in kilograms; $X_{3}$ is the subject's femur length; $\mathrm{X}_{4}$ is the subject's gender; $\mathrm{B}_{0}=\mathrm{Y}$ intercept; $\mathrm{B}=$ the slope associated with each of the specified parameters; $\varepsilon$ is the unexplained error.

The regression line was constructed by the theory of least squares method using statistical software by NCSS/ PASS Inc. (Kayesville, UT). A coefficient of determination $\left(\mathrm{R}^{2}\right)$ was calculated as a representation of the percentage of variability in PP attributed to the model using the Pearson product-moment procedure. Forward stepwise regression was used to retain only the terms that significantly $(P<0.05)$ contributed to the linearity of the model. This process was repeated for construction of the AP model.

\section{Results}

\section{Participant characteristics}

Of the 14 participants included in the study, 57.2\% (2 men, 6 women) completed the full 30-second protocol without resting. In the sample, 1 man and 1 woman used a walking aid on a regular basis, and 2 men and 1 woman reported at least one fall within the past 5 years.

\section{Performance testing: protocol selection and concentric power}

All participants achieved an uninterrupted 20 seconds of maximal chair rises. Discriminate analysis revealed that the number of stands performed in 20 seconds contributed more to the linearity of the predictive model than the number performed in 5 seconds or in 10 seconds. Therefore, in our model we used the number of chair rises performed in the first 20 seconds (of the 30 -second test), which on average was $9.8 \pm 3.5$.

The average concentric power per chair rise was $462.3 \pm 212 \mathrm{~W}\left(6.4 \pm 1.7 \mathrm{~W} \cdot \mathrm{kg}^{-1}\right)$ and ranged from $274.6 \mathrm{~W}$ $\left(4.5 \mathrm{~W} \cdot \mathrm{kg}^{-1}\right)$ to $930.7 \mathrm{~W}\left(10.5 \mathrm{~W} \cdot \mathrm{kg}^{-1}\right)$. Peak concentric power achieved during the test averaged $586.3 \pm 274.7 \mathrm{~W}$, or $8.2 \pm 2.3 \mathrm{~W} \cdot \mathrm{kg}^{-1}$. The average time at which the most powerful chair rise was performed was highly variable $(6.6 \pm 7.5$ seconds), and there was no significant relationship between the highest power produced among participants and the time at which maximum power was achieved $\left(\mathrm{R}^{2}=-0.25\right.$; $P>0.05)$. Similarly, the chair rise that produced the greatest power was highly variable, ranging from the first to the last chair rise performed. The mode for the chair rise that produced the greatest power among participants was repetition one (Figure 2).

\section{Regression analysis: peak and average chair-rise power}

Femur length and gender failed to contribute significantly to the regression models for PP or AP $\left(\mathrm{R}^{2}=0.006,0.003\right.$, and 0.004, respectively; $P>0.05$ ). Therefore, the resultant regression analysis consisted of the two remaining independent variables: 1) number of chair rises in 20 seconds; and 2) body weight. The subsequent analysis resulted in the following equations for AP and PP.

1. Average power $(\mathrm{W})=-504.845+10.793$ body weight $(\mathrm{kg})+21.603$ stands in $20 \mathrm{~s}\left(\mathrm{R}^{2}=0.784, P<0.01\right)$

2. Peak power $(\mathrm{W})=-715.218+13.915$ body weight $(\mathrm{kg})+33.425$ stands in $20 \mathrm{~s}\left(\mathrm{R}^{2}=0.811, P<0.01\right)$

The mean predicted AP per chair rise was $458 \pm 33 \mathrm{~W}$, which was similar to the mean power measured during the chair rise, $462.3 \pm 27 \mathrm{~W}$. Similarly, the mean predicted PP was $586.3 \pm 178.4 \mathrm{~W}$, nearly identical to the actual peak power, $586.3 \pm 30 \mathrm{~W}$. The predicted AP and PP values were strongly correlated to the actual AP and PP values computed using force plate and movement analysis data (AP: $\mathrm{R}=0.89$; Figure 3; PP: $\mathrm{R}=0.90$; Figure 4).

\section{Discussion}

Our simple regression equations developed during this pilot study can predict lower-body PP and AP using the first 20 seconds of a 30-second chair-rise test. A number of factors favor the use of the chair-rise power test over other power-testing methods. For example, the Nottingham power rig, ${ }^{7}$ currently considered the "gold standard" in such testing, measures the power produced against a flywheel during combined hip extension, knee extension, and ankle plantar flexion. However, little or no upper body movement occurs, and dynamic balance adjustments are not necessary because the test is performed in a sitting position. Also, since the Nottingham rig is not a weight-bearing test, the kinetic chain 


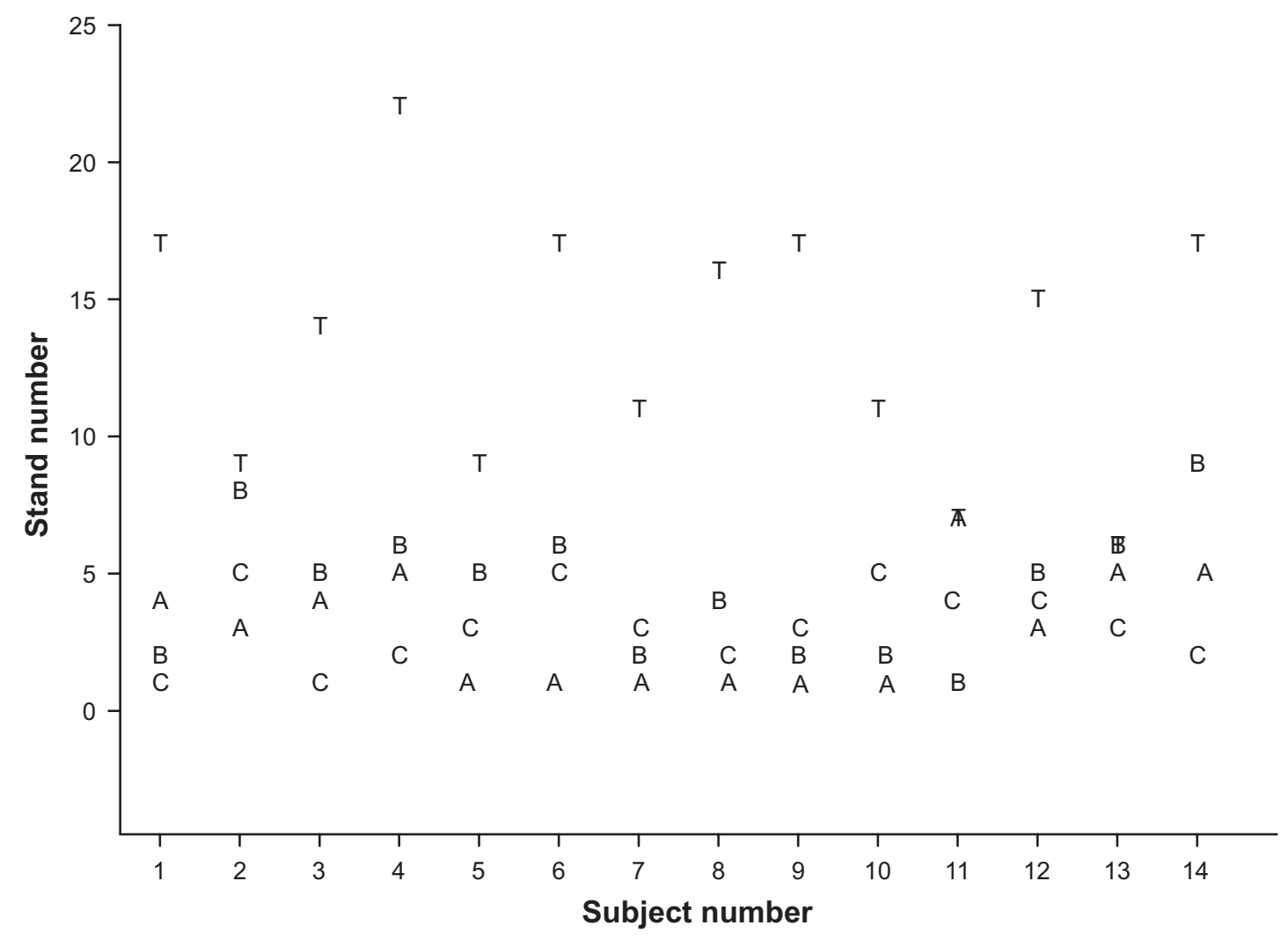

Figure 2 Graphic representation of the most powerful (A), second most powerful $(\mathbf{B})$, and third most powerful $(\mathbf{C})$ repetition and total $(\mathrm{T})$ repetitions.

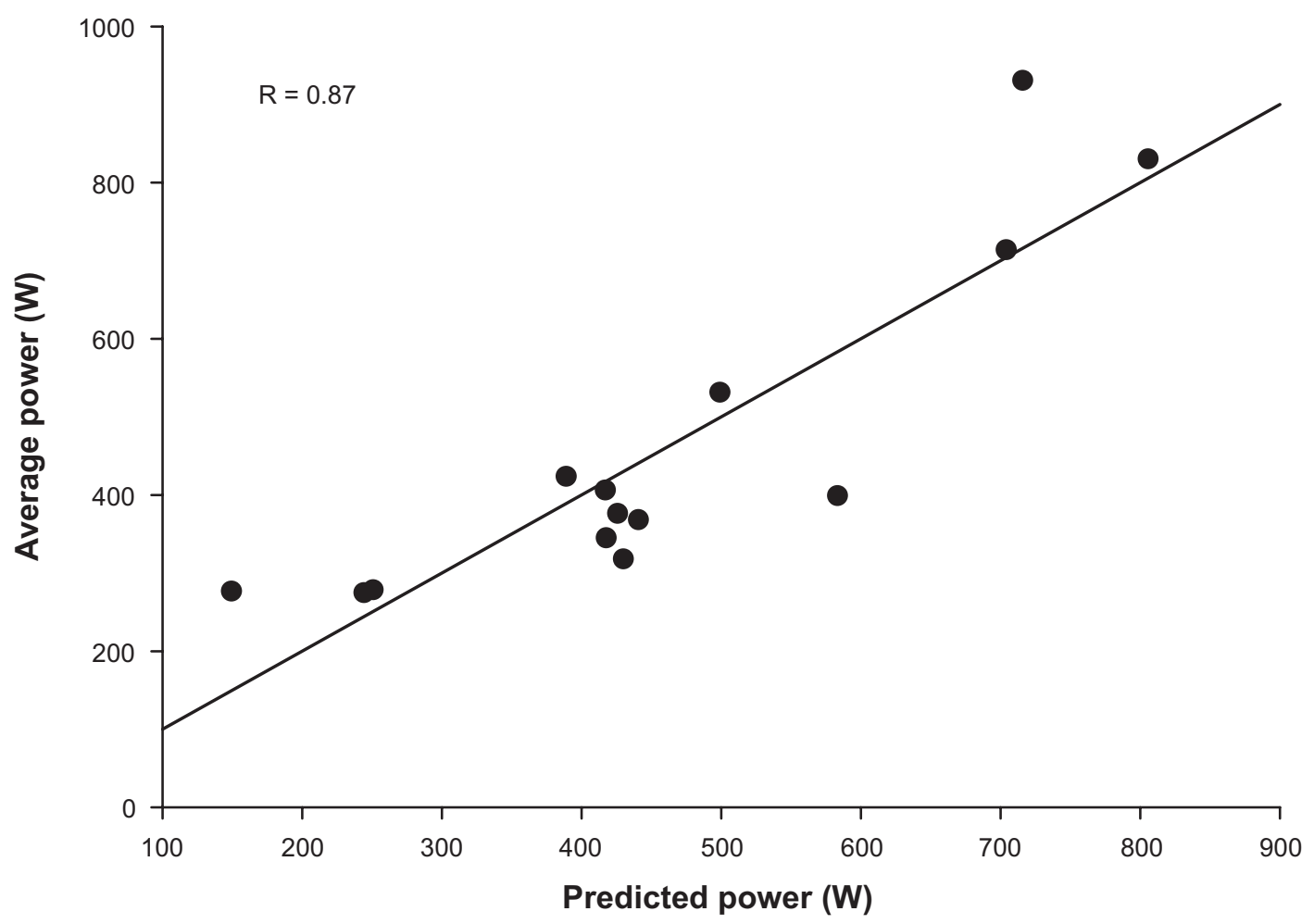

Figure 3 Plot of measured average power per chair rise versus average power predicted using our predictive equation. Predicted average power strongly correlated with actual average power $(R=0.89)$. 


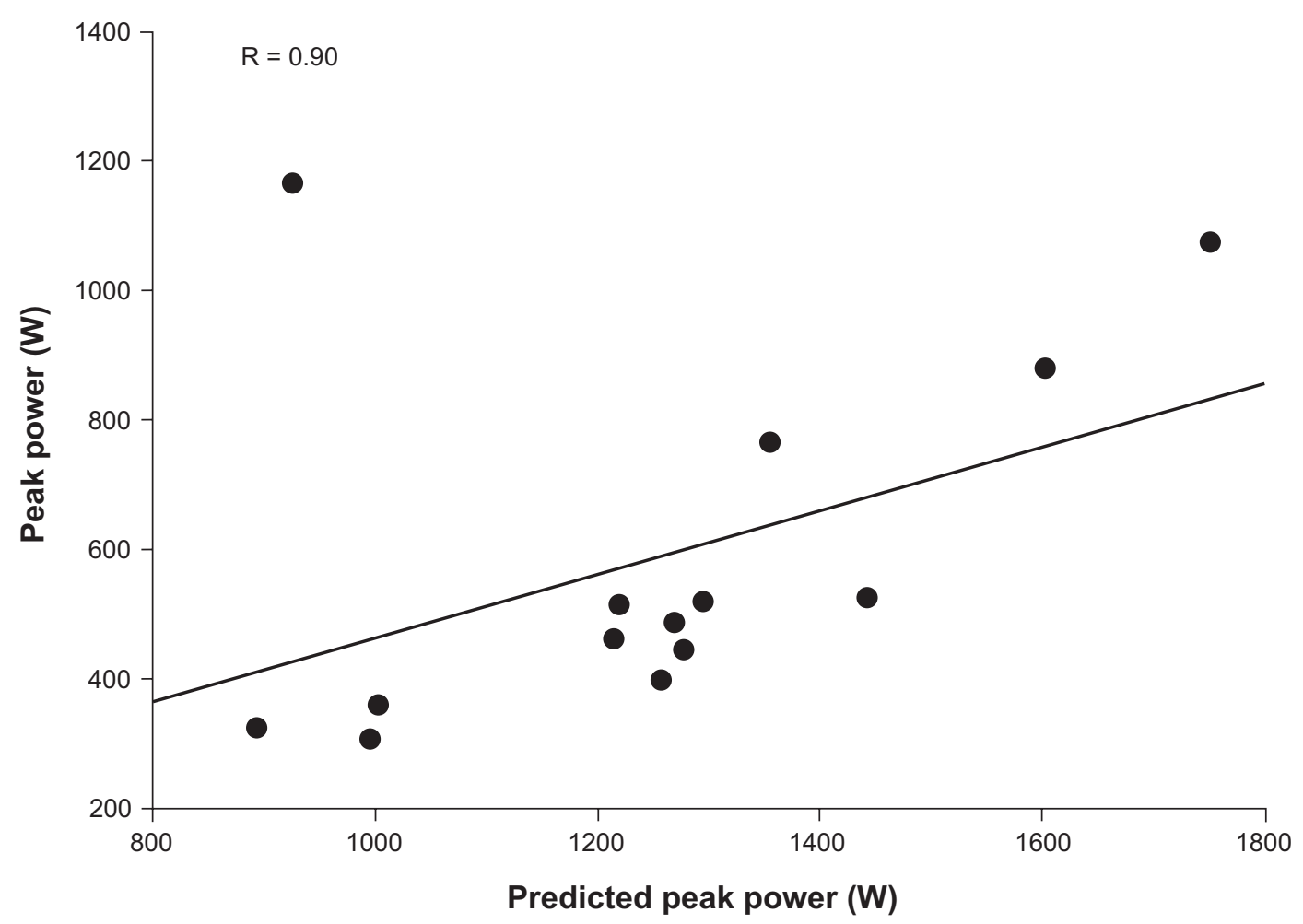

Figure 4 Plot of measured peak power across all chair rises versus peak power predicted using our predictive equation. Predicted peak power strongly correlated with actual peak power $(R=0.90)$.

of movement is dissimilar to most ADLs, and power outputs may not be correlated well with those produced during typical functional tasks. ${ }^{26,27}$ In contrast, the chair-rise test uses upperand lower-body musculature, requires continuous balance adjustments during sit-to-stand transitions, and employs a motor pattern commonly used in daily living.

The five-chair-rise test has been used to assess functionality in older persons. ${ }^{28}$ However, Lindemann and colleagues $^{26}$ reported a weak association between power and the five-chair-rise test and suggested that this was due to a ceiling effect during five stands. During our testing sessions, $35.7 \%$ of our participants achieved PP after the fifth chair rise, suggesting that the five-chair-rise test would not have measured functional power in these participants. Additionally, the 20-second time period reduces the potential for a ceiling effect because it is long enough to allow PP to be reached and short enough to allow all participants to complete the test.

Fleming et al. ${ }^{29}$ quantified power during a single maximal-speed stand-and-sit. They used the rate of force change during the stand to compute peak power. Although these values may be associated with concentric and eccentric power during a chair rise, methodological questions remain. For example, the force produced during a chair rise is not related solely to the vertical force applied. Other factors must be considered, such as the forces about the joints during different phases of the stand, including those during hip flexion at start of movement, hip and knee extension at seat-off, and trunk extension and maintenance of balance throughout the movement. ${ }^{31}$ During our testing we noted a decrease in ground reaction force during the initial hipflexion phase while the pelvis was tilted anteriorly and the lower back came away from the seat rest. ${ }^{26}$ This observation was confirmed by EMG data collected during the test, which showed that the decrease in force during this initial movement occurred concurrently with hip-flexor activation (data not shown). Other fluctuations in the recorded ground reaction force were likely indicative of the calf and peroneal muscles acting on the ankle joint to stabilize stance between chair rises. ${ }^{32}$ Unlike the measurement by Fleming et al. ${ }^{29}$ our testing used the product of movement velocity and simultaneous ground reaction force to measure power.

Despite the strengths of our study, which include its closer correlation with ADLs, ${ }^{1-3}$ ease of application in a clinical setting, and low cost, there are limitations. First, the regression equations were developed using data from 14 independent-living participants. Future studies should evaluate the robustness of these power equations with a larger 
group with greater diversity. For example, femur length may be a contributing factor in our equation in a sample with more divergent height measurements. Additionally, incorporating less-independent participants, especially those with greater probability of falls where postural sway may become an important contributing factor, may require a modification of the equation.

The second limitation to our study is the lack of comparisons to existing power tests. While our equations are derived from direct measurements of power, the association between these power equations and other tests of lower-body power should be examined to establish concurrent validity and examine the concept of power specificity as it relates to different functional movements. ${ }^{33}$ Correlations should be examined between power computed from this test and from other measures of functional debility and physical vulnerability in elderly participants.

A final weakness of this study relates to the "basement effect," which exists for participants weighing less than $50 \mathrm{~kg}$ who cannot complete more than one chair stand. In this instance, the predicted power would be negligible and potentially less than zero. However, the y-intercept of our equation for peak power suggests that the power required to perform a single chair rise is approximately $4 \mathrm{~W} \cdot \mathrm{kg}^{-1}$; therefore, a participant weighing less than $50 \mathrm{~kg}$ who performs only one chair rise may have less than the predicted power necessary to perform the test.

\section{Conclusion}

In conclusion, the present study provides equations that allow the measurement of concentric AP and PP using a simple chair-rise protocol, which constitutes a simple, cost-effective, and efficient power test accessible to a large segment of the older population. The test requires only an armless chair, a stopwatch, and an average testing period of less than 10 minutes including a warm-up, demonstration, and two trials separated by five minutes of recovery. Because of the strong link between lower-body power and functional independence in the elderly, ${ }^{1-3}$ the equations tested in this study can be useful in predicting physical vulnerability and progressive debility.

\section{Acknowledgment}

The authors would like to thank Mr Steven E Hwang for his help in the preparation of this article.

\section{Disclosure}

The authors report no conflicts of interest in this work.

\section{References}

1. Skelton DA, Greig CA, Davies JM, Young A. Strength, power and related functional ability of healthy people aged 65-89 years. Age Ageing. 1994;23:371-377.

2. Skelton DA, Kennedy J, Rutherford OM. Explosive power and asymmetry in leg muscle function in frequent fallers and non-fallers aged over 65. Age Ageing. 2002;31:119-125.

3. Spirduso WW. Balance, posture, and locomotion. Physical Dimensions of Aging. Champaign, IL: Human Kinetics. 1995;155-183.

4. Bassey EJ, Fiatarone MA, O’Neill EF, Kelly M, Evans WJ, Lipsitz LA. Leg extensor power and functional performance in very old men and women. Clin Sci (London). 1992;82:321-327.

5. Booth FW, Weeden SH, Tseng BS. Effect of aging on human skeletal muscle and motor function. Med Sci Sports Exerc. 1994; 26:556-560.

6. Baker D, Nance S, Moore M. The load that maximizes the average mechanical power output during jump squats in power-trained athletes. J Strength Cond Res. 2001;15:92-97.

7. Bassey EJ, Short AH. A new method for measuring power output in a single leg extension: feasibility, reliability and validity. Eur $J \mathrm{Appl}$ Physiol. 1990;60:385-390.

8. De Vito G, Bernardi M, Forte R, Pulejo C, Macaluso A, Figura F. Determinants of maximal instantaneous muscle power in women aged 50-75 years. Eur J Appl Physiol. 1998;78:59-64.

9. Doherty TJ. Invited review: aging and sarcopenia. J Appl Physiol. 2003;95:1717-1727.

10. Evans W. Functional and metabolic consequences of sarcopenia. J Nutr. 1997;127:998S-1003S.

11. Fielding RA, LeBrasseur NK, Cuoco A, Bean J, Mizer K, Fiatarone Singh MA. High-velocity resistance training increases skeletal muscle peak power in older women. J Am Geriatr Soc. 2002;50:655-662.

12. Grassi B, Cerretelli P, Narici MV, Marconi C. Peak anaerobic power in master athletes. Eur J Appl Physiol. 1991;62:394-399.

13. Harman EA, Rosenstein MT, Frykman PN, Rosenstein RM, Kraemer WJ. Estimation of human power output from vertical jump. J Strength Cond. Res. 1991;5:116-120.

14. Huskey T, Mayhew JL, Ball TE, Arnold MD. Factors affecting anaerobic power output in the Margaria-Kalamen test. Ergonomics. 1989;32:959-965.

15. Kaneko M, Fuchimoto T, Toji H, Suei K. Training effect of different loads on the force-velocity relationship and mechanical power output in human muscle. Scand J Sports Sci. 1983;5:50-55.

16. Margaria R, Aghemo P, Rovelli E. Measurement of muscular power (anaerobic) in man. J Appl Physiol. 1966;21:1662-1664.

17. Mayhew JL, Hampton BK, Armstrong W. Task specificity among power tests in college males. Kansas AHPER J. 1981;49:5-7.

18. Mayhew JL, Piper FC, Schwegler TM, Ball TE. Effects of motor dexterity and acceleration momentum on anaerobic power production. Percept Mot Skills. 1986;64:1055-1060.

19. Osternig LR. Optimal isokinetic loads and velocities producing muscular power in human subjects. Arch Phys Med Rehabil. 1975;56:152-155.

20. Signorile JF, Sandler D, Kempner L, Stanziano D, Ma F, Roos BA. The ramp power test: a new method of power assessment during a functional task for older individuals. J Gerontol. 2007;62A:1266-1273.

21. Bialoszewski D, Slupik A, Lewczuk E, Gotlib J, Mosiolek A, Mierzwinska A. Incidence of falls and their effect on mobility of individuals over 65 years of age relative to their place of residence. Ortop Traumatol Rehabil. 2008;10:441-448.

22. Henwood TR, Taaffe DR. Improved physical performance in older adults undertaking a short-term programme of high-velocity resistance training. Gerontology. 2005;51:108-115.

23. Signorile JF, Carmel MP, Czaja SJ, et al. Differential increases in average isokinetic power by specific muscle groups of older women due to variations in training and testing. J Gerontol A Biol Sci Med Sci. 2002;57A: M683-M690.

24. Rikli RE, Jones CJ. Development and validation of a functional fitness test for community-residing older adults. J Aging Phys Act. 1999; 7:129-161 
25. Eames MHA, Cosgrove A, Baker R. Comparing methods of estimating the total body center of mass in the three dimensions in normal and pathological gaits. Hum Mov Sci. 1998;18:637-646.

26. Lindemann U, Claus H, Stuber M, et al. Measuring power during the sit-to-stand transfer. Eur J Appl Physiol. 2003;89:466-470.

27. Pearson SJ, Harridge SD, Grieve DW, Young A, Woledge RC. A variable inertial system for measuring the contractile properties of human muscle. Med Sci Sports Exerc. 2001;33:2072-2076.

28. Guralnik JM, Simonsick EM, Ferrucci L, et al. A short physical performance battery assessing lower extremity function: association with self-reported disability and prediction of mortality and nursing home admission. J Gerontol Med Sci. 1994;49:M85-M94.

29. Fleming BE, Wilson DR, Pendergast DR. A portable, easily performed muscle power test and its association with falls by elderly persons. Arch Phys Med Rehabil. 1991;72:886-889.
30. Hirschfeld H, Thorsteinsdottir M, Olsson E. Coordinated ground forces exerted by buttocks and feet are adequately programmed for weight transfer during sit-to-stand. J Neurophysiol. 1999;82:3021-3029.

31. Kralj A, Jaeger RJ, Munih M. Analysis of standing up and sitting down in humans: definitions and normative data presentation. J Biomech. 1990;23:1123-1138.

32. Roebroeck ME, Doorenbosch CAM, Harlaar J, Jacobs R, Lankhorst GJ. Biomechanics and muscular activity during sit-to-stand transfer. Clin Biomechan. 1994;9:235-244.

33. Cuoco A, Callahan DM, Sayers S, Frontera WR, Bean J, Fielding RA. Impact of muscle power and force on gait speed in disabled older men and women. J Gerontol A Biol Sci Med Sci. 2004;59:1200-1206.
Clinical Interventions in Aging

\section{Publish your work in this journal}

Clinical Interventions in Aging is an international, peer-reviewed journal focusing on evidence-based reports on the value or lack thereof of treatments intended to prevent or delay the onset of maladaptive correlates of aging in human beings. This journal is indexed on PubMed Central, MedLine, the American Chemical Society's 'Chemical

\section{Dovepress}

Abstracts Service' (CAS), Scopus and the Elsevier Bibliographic databases. The manuscript management system is completely online and includes a very quick and fair peer-review system, which is al easy to use. Visit http://www.dovepress.com/testimonials.php to read real quotes from published authors. 